Computer Optics and Nanophotonics

\title{
DIFFRACTIVE AXICONS TO INCREASE THE EFFICIENCY OF SOLAR CELLS
}

\author{
V.V. Podlipnov ${ }^{1,2}$, A.P. Porfirev ${ }^{1,2}$, S.A. Degtyarev ${ }^{1,2}$, S.N. Khonina ${ }^{1,2}$ \\ ${ }^{1}$ Samara National Research University, Samara, Russia \\ ${ }^{2}$ Image Processing Systems Institute - Branch of the Federal Scientific Research Centre "Crys- \\ tallography and Photonics" of Russian Academy of Sciences, Samara, Russia
}

\begin{abstract}
We propose to integrate diffractive axicons into solar cells as a light trap for increasing of light absorbing efficiency. Experimental work and numerical simulations are provided in this paper. The experimental measurements of light-to-electricity conversion efficiency are conducted using a laser with tunable wavelength.
\end{abstract}

Keywords: solar cell, diffraction grating, axicon, light trapping.

Citation: Podlipnov VV, Porfirev AP, Degtyarev SA, Khonina SN. Diffractive Axicons to Increase the Efficiency of Solar Cells. CEUR Workshop Proceedings, 2016; 1638: 103-110. DOI: 10.18287/1613-0073-2016-1638-103-110

\section{Introduction}

The latest days increasing of efficiency and decreasing of the cost can be noticed in the solar power engineering development. It is providing a significant growth of photovoltaic energy production and solar cells manufacturing $[1,2]$.

Thus, solar cells efficiency increasing methods development is a relevant problem at present. There are two problem classes of light-to-electricity conversion efficiency. The first is the problem of efficiency increasing of light transportation to an active semiconductor (optical efficiency problem). And the second one is the problem of efficiency increasing of the delivered light conversion into electric current (quantum efficiency problem).

There is a review in the paper [3] where basic methods of optical efficiency increasing problem solving are shown. Among the mentioned methods we can emphasize a method which consists of using of additional optical elements and devices such as mirrors and diffractive and refractive optical elements for high-performance concentration and delivering of light towards a solar cell. This approach makes it possible to decrease the cost of electric energy generation due to significant decreasing the solar element area $[4,5]$.

After analysis of optical efficiency increasing methods we can conclude that the integration of diffractive gratings and solar cells is the inexpensive and appropriate approach to efficient solar cells manufacturing. 
These elements can be manufactured with inexpensive nanoimprint lithography [6] and following plasma-chemical etching which are well compatible with microelectronic technology and classical silicon solar cells manufacturing technology.

However, despite the great amount of papers devoted this area, there are not enough researches about using Fresnel diffractive lenses and diffractive axicons as a light traps for solar cells. In the most of papers these diffractive devices are used as thinfilmy auxiliary optical elements which are light concentrators [7].

Thus, it seems to be relevant to use arrays of diffractive microaxiconical structures which are etched in front surface of a solar cell. These diffractive structures serve at the same time as an antirelfecting and focusing structure, also it scatters light inside of active semiconductor due to diffraction and hence elongates the optical path and increases quantum efficiency of light-to-electricity conversion. The Kirchhoff diffraction integral is used to calculate the spatial distribution of the optical field in the focal region of an optical element in the paper [8]. Numerical simulation comparison of two types diffractive axicons - linear and logarithmic - under characteristics of formed distributions of intensity both longitudinal, and cross section is conducted the in the paper [9].

In this work we describe the research of diffractive axicons utilizing opportunity for solar cells efficiency increasing.

\section{$2 \quad$ Axicon and solar cell combination}

It is well known that diffractive axicon transforms initial plane wave front into conical wave surface which converges towards the optical axis $\mathrm{z}$ at angle $\alpha$ (fig. 1). Thus, onaxis light segment which has a full width at a half maximum of равна $0,36 \lambda / \mathrm{NA}$ ( $\lambda$ is a wavelength, $\mathrm{NA}=\sin \alpha-$ numerical aperture of the axicon), that is significantly less than Airy disk diameter which is produced with a lens with the same numerical aperture [10-14]. Moreover, a depth of focus is much greater for an axicon than for a lens even for high numerical apertures $[13,14]$. This fact makes axicons to be useful for those systems where it is difficult to precisely adjust all distances. It is especially important in medical $[15,16]$ and metrological $[17,18]$ applications.

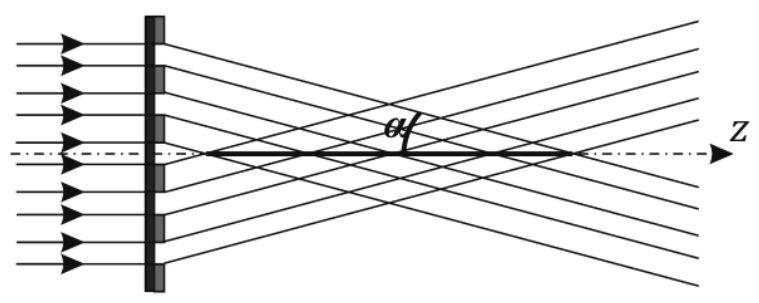

Fig. 1. Working principle of diffractive axicon 
Notice that if the period of a diffractive axicon is less than the wavelength (subwavelength axicons) [19-21], the axicon transforms the most part of initial illumination into scattering waves which propagate perpendicular to the optical axis, that is the light propagates along the surface of the element.

In figure 2 there are calculated energy patterns of light propagation through axicons with high numerical aperture. In this figure we can see that the length of on-axis light segment is becoming shorter and light is scattered at higher angles with increasing of numerical aperture. In addition, from figure 3 we can derive that light scattering intensifies with increasing of the wavelength.
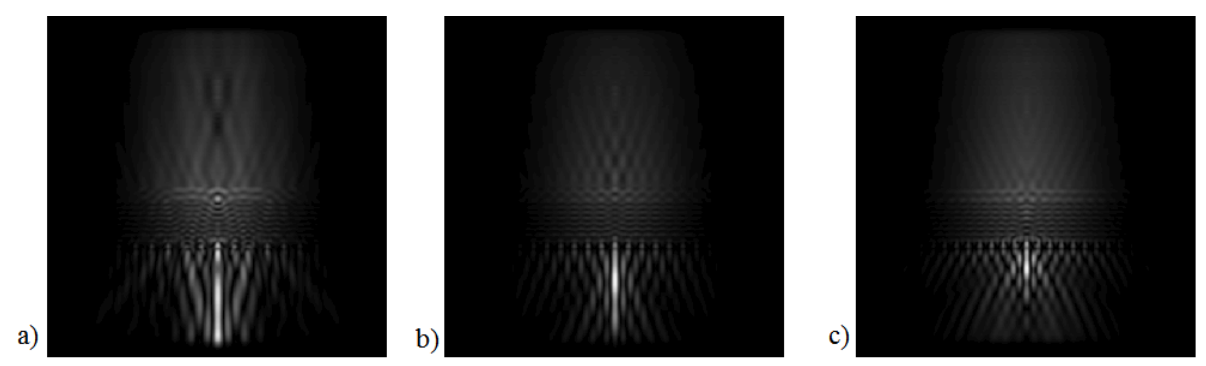

Fig. 2. Calculated intensity patterns whereas light propagates through the axicon with various numerical aperture a) $\mathrm{NA}=0.71$, b) $\mathrm{NA}=0.87$, c) $\mathrm{NA}=0.95$

a)
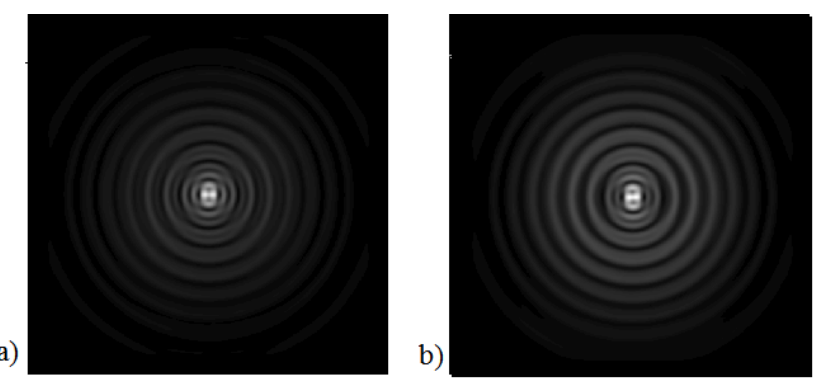

Fig. 3. Transverse patterns of light diffraction on the axicon near the axicon surface for different wavelength of incident light: a) $532 \mathrm{~nm}$, b) $633 \mathrm{~nm}$

Thus, axicons can increase efficiency of light capturing due to high-angle scattering of normally impinging light.

In figure 4 we propose a way of combining a diffractive axicon and a solar element. The axicon is placed at the front surface of the solar cell. The axicon scatters impinging light and hence it elongates the optical path of the rays. It must increase the possibility of photon capturing inside the active medium. 


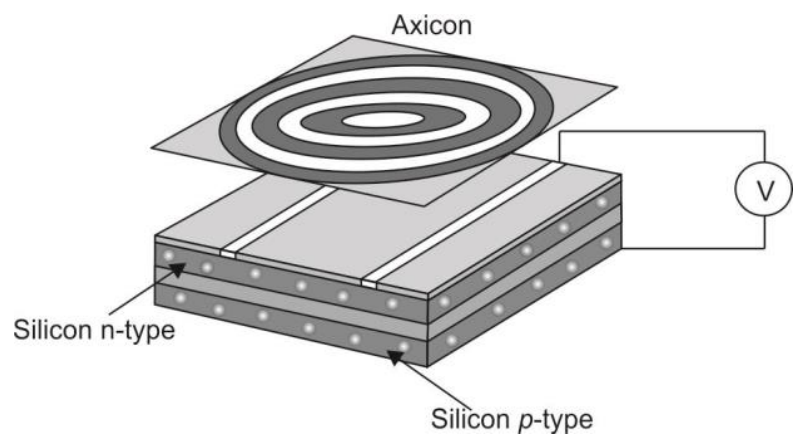

Fig. 4. Combination of diffractive axicon and a solar element Figure 4. Combination of a solar element and axicon

\section{$3 \quad$ Numerical simulations}

This section describes conducted numerical simulation of the light absorbing in solar cell active semiconductor. The simulation is based on Helmholtz equations solving with finite element method using Comsol Multiphysics software. In this paper we consider only $2 \mathrm{~d}$ model.

General view of simulation layout is shown in figure 5. Plane wave illuminates the solar cell element from above then light passes through the thin glass layer, active silicon medium and then reflects from the bottom silver layer. The thickness of the silicon layer is $1 \mu \mathrm{m}$, silver layer is $40 \mathrm{~nm}$ thick, and glass substrate is $1.8 \mu \mathrm{m}$ thick. Diffractive grating relief height is $300 \mathrm{~nm}$.

Plane wave origin is set in the top side of calculation domain. Lateral sides satisfy Bloch conditions which provide periodicity of the grating. Thus, the calculation domain is one period of diffractive grating.

Intensity distribution from figure 5a corresponds to standing wave.

Absorption spectrum is calculated as a following way. We calculate intensity distribution in all defined domain for a list of wavelength from $300 \mathrm{~nm}$ to $900 \mathrm{~nm}$ with the step of $5 \mathrm{~nm}$. Then, for every wavelength we integrate the intensity in the area of active domain. The meaning of mentioned above integral is taken as a meaning of absorption.

In the figure 6 there are absorption spectrums for a solar sell that is covered by glass plate and also glass diffractive grating.

We can see, that spectrums significantly differ from one another. Diffraction grating makes the absorption about ten times higher for the wavelength of $585 \mathrm{~nm}$. This fact can be utilized for increasing of solar cell efficiency.

We should remember that a solar cell is illuminated with multispectral natural light with complex spectrum profile. Thus, it has to be noticed that for one wavelength diffractive grating increase the efficiency, but it does not have to work for another wavelength. Hence, the research of multispectral absorption increasing with grating needs to be provided more careful. 


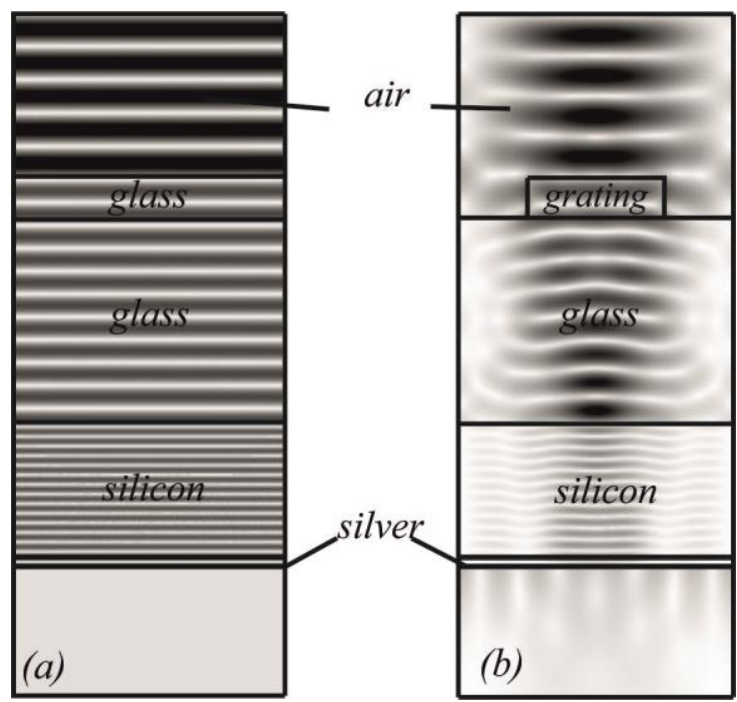

Fig. 5. Intensity distribution whereas plane wave propagates through the solar cell covered by a) glass substrate and b) diffractive grating
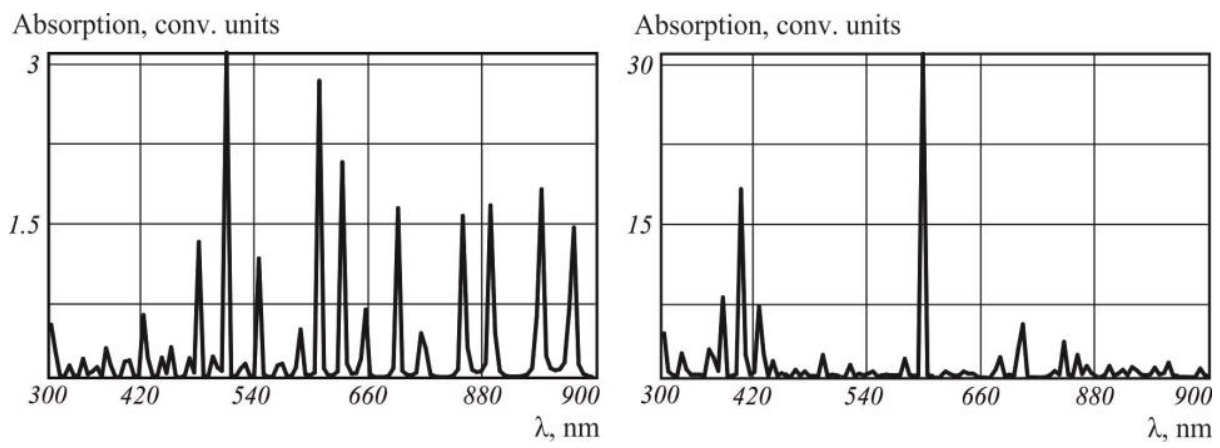

Fig. 6. Absorption spectrums of the element a) without and b) with grating

\section{Experimental measurements}

We set an optical layout which is shown at figure 7 . We use laser with a tunable wavelength EKSPLA NT242 as a light origin. This laser can produce a beam with a wavelength from the range from $195 \mathrm{~nm}$ to $2600 \mathrm{~nm}$. The mirror M reflects laser and redirects it into the combination of diffractive axicon and the solar cell. The axicon is placed to the front surface of the solar cell. Using electro-multimeter $\mathrm{V}$ we measure a current which is generated with the solar cell. 


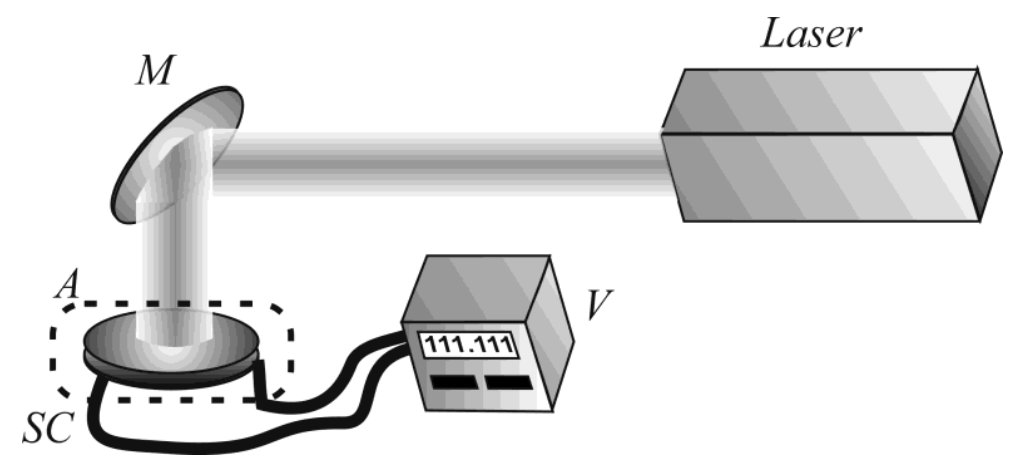

Fig. 7. Experimental setup for measuring of voltage in solar cell circuit. Laser - laser with a tunable wavelength EKSPLA NT242, M - mirror, A - diffractive axicon, SC - solar element, $\mathrm{V}$ - electro-multimeter

In conducted experiments we measure a current in the solar cell circuit combining with a glass substrate at the first series of measurements and with diffractive axicon with period of $2 \mu \mathrm{m}$ in the second series of measurements.

Scanning Electronic Microscopic photograph of the axicon is shown in the figure 8a, in figure $8 \mathrm{~b}$ we show intensity pattern at the distance of few microns from the axicons surface under illumination of light with the wavelength of $633 \mathrm{~nm}$. Intensity distribution from figure $8 \mathrm{~b}$ was experimentally measured with near-field microscope. The axicon has numerical aperture $\mathrm{NA}=0.32$.

a)

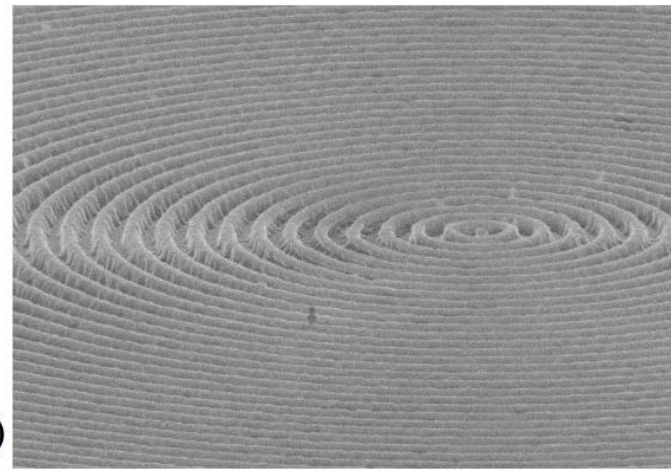

\section{b)}

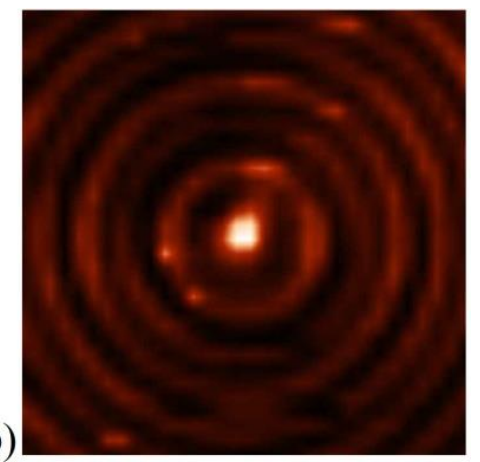

Fig. 8. General view of central part of the axicon a) and experimentally measured diffractive intensity pattern in near field

The measured meanings are shown in table 1. From the table we can derive that combination of the solar cell and axicon provides voltage increasing in the range from 400 $\mathrm{nm}$ to $800 \mathrm{~nm}$. 
Table 1. Dependence of solar cell circuit voltage versus the initial illumination wavelength

\begin{tabular}{lcc}
\hline \multirow{2}{*}{$\begin{array}{c}\text { Initial illumination } \\
\text { wavelength, } \mathbf{n m}\end{array}$} & \multicolumn{2}{c}{ Voltage in solar cell circuit, V } \\
\cline { 2 - 4 } & $\begin{array}{c}\text { Combination of solar cell } \\
\text { and glass substrate }\end{array}$ & $\begin{array}{c}\text { Combination of solar cell } \\
\text { and diffractive axicon }\end{array}$ \\
$\mathbf{4 0 0}$ & 0.35 & $\mathbf{0 . 5 5}$ \\
$\mathbf{4 5 0}$ & 0.86 & $\mathbf{1 . 0 0}$ \\
$\mathbf{5 0 0}$ & 0.99 & $\mathbf{1 . 0 3}$ \\
$\mathbf{5 5 0}$ & 1.06 & $\mathbf{1 . 0 4}$ \\
$\mathbf{6 0 0}$ & 1.01 & $\mathbf{1 . 0 3}$ \\
$\mathbf{6 5 0}$ & 0.95 & $\mathbf{0 . 9 4}$ \\
$\mathbf{7 0 0}$ & 0.44 & $\mathbf{0 . 5 3}$ \\
\hline $\mathbf{7 5 0}$ & 0.33 & $\mathbf{0 . 3 9}$ \\
\hline $\mathbf{8 0 0}$ & $\mathbf{0 . 0 4}$ & $\mathbf{0 . 0 4}$ \\
\hline
\end{tabular}

\section{Conclusions}

Conducted experiments show advantages of diffractive axicon using for increasing of solar cells efficiency.

Numerical simulations of absorption spectrum qualitatively show benefits of diffractive axicon using for absorption increasing.

In future we are planning to provide more detailed research of dependence of solar cells quantum efficiency on different periods (including subwavelength) of diffractive axicons which are combined with the solar element.

\section{Acknowledgements}

This work was funded by Russian Scientific Fund (grant No. 14-31-00014).

\section{References}

1. Alferov ZhI, Andreev VM, Rumyantcev VD. Solar Photovoltaics: Trends and Prospects. Semiconductors, 2004; 38(8): 899-908.

2. Kozukov DA, Tsygankov BK. Analysis of advantages and rates of development of solar photovoltaics. Innovative Science, 2015; 8(2): 38-41 [in Russian].

3. Wehrspohn RB, Rau U, Gombert A. Photon Management in Solar Cells. John Wiley \& Sons, 2015.

4. Andreev VM, Davidyuk NYu, Ionova EA, Rumyantsev VD. Photovoltaic modules with cylindrical waveguides in a system for the secondary concentration of solar radiation. Technical Physics, 2013; 58(9): 1323.

5. Barnett A. Very high efficiency solar cell modules. Progress in Photovoltaics: Research and Applications, 2009; 17(1): 75-83. 
6. Hauser H, Mellor A, Guttowski A, Wellens C, Benick J, Muller C, Hermle M, Blasi B. Diffractive backside structures via nanoimprint lithography. Energy Procedia, 2012; 27: 337-342.

7. Chemisana D. Building integrated concentrating photovoltaics: a review. Renewable and Sustainable Energy Reviews, 2011; 15(1): 603-611.

8. Golub MA, Kazanskii NL, Sisakyan IN, Soifer VA, Kharitonov SI. Diffraction calculation for an optical element which focuses into a ring. Optoelectronics, Instrumentation and Data Processing, 1987; (6): 7-14.

9. Khonina SN, \& Balalayev SA. The comparative analysis of the intensity distributions formed by diffractive axicon and diffractive logarithmic axicon. Computer Optics, 2009; 33(2): 162-174.

10. Kalosha VP, Golub I. Toward the subdiffraction focusing limit of optical superresolution. Optics Letters, 2007; 32: 3540-3542.

11. Khonina SN, Volotovsky SG. Fracxicon - diffractive optical element with conical focal domain. Computer Optics, 2009; 33(4): 401-411.

12. Khonina SN, Ustinov AV, Volotovsky SG, Kovalev AA. Calculation of diffraction of the linearly-polarized limited beam with uniform intensity on high-aperture binary microaxicons in a near zone. Computer Optics, 2010; 34(4): 443-460.

13. Khonina SN, Serafimovich PG, Savelyev DA, Pustovoi IA. Diffraction at binary microaxicons in the near field. Journal of Optical Technology, 2012; 79(10): 626-631.

14. Khonina SN, Degtyarev SA. A longitudinally polarized beam generated by a binary axicon. Journal of Russian Laser Research, 2015; 36(2): 151-161.

15. Leitgeb RA, Villiger M, Bachmann AH, Steinmann L, Lasser T. Extended focus depth for Fourier domain optical coherence microscopy. Optics Letters, 2006; 31(16): 2450-2452.

16. Lee K-S, Rolland JP. Bessel beam spectral-domain high-resolution optical coherence tomography with micro-optic axicon providing extended focusing range. Optics Letters, 2008; 33(15): 1696-1698.

17. Kotlyar VV, Skidanov RV, Khonina SN. Non-contact precision measurement of linear displacement with DOE forming fashion Bessel. Computer Optics, 2001; 21: 102-104 [in Russian].

18. Wang K, Zeng L, Yin Ch. Influence of the incident wave-front on intensity distribution of the nondiffracting beam used in large-scale measurement. Optics Communication, 2003; 216: 99-103.

19. Khonina SN, Nesterenko DV, Morozov AA, Skidanov RV, Pustovoy IA. Experimental research of diffraction of an linearly-polarized gaussian beam by binary microaxicon with the period close to wavelength, Computer Optics, 2011; 35(1): 11-21.

20. Khonina SN, Karpeev SV, Alferov SV, Savelyev DA, Laukkanen J, Turunen J. Experimental demonstration of the generation of the longitudinal E-field component on the optical axis with high-numerical-aperture binary axicons illuminated by linearly and circularly polarized beams. Journal of Optics, 2013; 15: 085704-085711.

21. Khonina SN, Savelyev DA. High-aperture binary axicons for the formation of the longitudinal electric field component on the optical axis for linear and circular polarizations of the illuminating beam. Journal of Experimental and Theoretical Physics, 2013; 117(4): 623630 . 